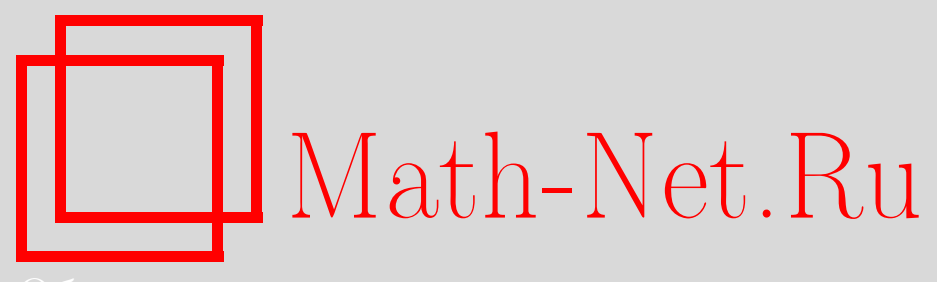

Л. А. Ляпков, Б. А. Севастьянов, Предельное распределение вероятностей перманента случайной матрицы в поле GF $(p)$, Дискрет. матем., 1996, том 8, выпуск 2, 3-13

DOI: https://doi.org/10.4213/dm522

Использование Общероссийского математического портала Math-Net.Ru подразумевает, что вы прочитали и согласны с пользовательским соглашением http: //www.mathnet.ru/rus/agreement

Параметры загрузки:

IP : 52.23 .180 .231

26 апреля 2023 г., 18:25:53

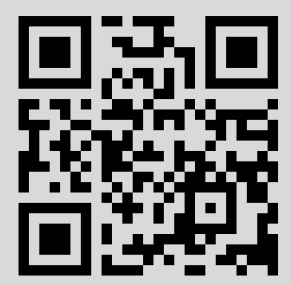




\section{Предельное распределение вероятностей перманента случайной матрицы в поле $\mathrm{GF}(p)$}

(C) 1996 г. Л. А. Ляпков, Б. А. Севастьянов

Показано, что перманент $\operatorname{per}\left(A_{n m}\right)$ случайной $n \times m$ матрицы $A_{n m}$ с әлементами из поля $\mathrm{GF}(p)$ и независимыми строками имеет предельное при $n \rightarrow \infty$ распределение вероятностей $\left\{p_{k}\right\}, k=0,1,2, \ldots, p-1$, вида

$$
p_{k}=\rho_{m} \delta_{k 0}+\left(1-\rho_{m}\right) / p
$$

где $\delta_{k 0}-$ символ Кронекера. Эти вероятности при каждом $m$ совпадают с распределением вероятностей некоторой функции от независимых и равномерно распределенных на $\mathrm{GF}(p)$ случайных величин.

Работа выполнена при поддержке Российского фонда фундаментальных исследований, проект 93-011-1443.

\section{1. Постановка задачи}

Перманент матрицы $A_{n m}=\left\|\alpha_{i j}\right\|$ с $n$ строками и с $m$ столбцами, $n \geqslant m$, определяется формулой

$$
\operatorname{per}\left(A_{n m}\right)=\sum_{\left(i_{1}, i_{2}, \ldots, i_{m}\right)} \alpha_{i_{1} 1} \alpha_{i_{2} 2} \ldots \alpha_{i_{m} m}
$$

где сумма берется по всем наборам $\left(i_{1}, i_{2}, \ldots, i_{m}\right)$ попарно различных индексов. Мы будем предполагать, что $\alpha_{i j}$ являются әлементами поля $\mathrm{GF}(p)$. В этом случае перманент принимает значения $0,1,2, \ldots, p-1$. Считая матрицу $A_{n m}$ случайной, найдем предельное распределение вероятностей $\mathbf{P}\left\{\operatorname{per}\left(A_{n m}\right)=k\right\}$, $k=0,1,2, \ldots, p-1$, при $n \rightarrow \infty$ и постоянном $m$. Сначала введем некоторые определения и обозначения, которыми будем далее пользоваться. Знаки + и $\sum$ будут, как правило, означать суммы по модулю $p$ (т.е. суммы в поле $\mathrm{GF}(p)$ ). Из контекста будет ясно, когда сумма понимается в обычном смысле. Мощность конечного множества $B$ будем обозначать $|B|$. В лемме 1 устанавливается вспомогательный результат о числе связных графов. В основной теореме $1 \operatorname{per}\left(A_{n m}\right)$ выражается с помощью некоторых функций от сумм $\sum_{i=1}^{n} \alpha_{i j}, \sum_{i=1}^{n} \alpha_{i j_{1}} \alpha_{i j_{2}}, \ldots$, $\sum_{i=1}^{n} \alpha_{i j_{1}} \alpha_{i j_{2}} \ldots \alpha_{i j_{p}}$. В лемме 2 устанавливается предельное распределение для 
вспомогательной цепи Маркова. В теореме 2 в достаточно общих условиях доказывается, что вероятности

$$
p_{m k}=\lim _{n \rightarrow \infty} \mathbf{P}\left\{\operatorname{per}\left(A_{n m}\right)=k\right\}, \quad k=0,1,2, \ldots, p-1,
$$

определяются распределением вероятностей некоторых функций от независимых и равномерно распределенных на $\operatorname{GF}(p)$ случайных величин $\xi_{j_{1}}, \xi_{j_{1} j_{2}}$, $\xi_{j_{1} j_{2} j_{3}}, \ldots, \xi_{j_{1} j_{2} \ldots j_{p}}, 1 \leqslant j_{1}<j_{2}<\ldots<j_{p} \leqslant m$

\section{2. Лемма о неразложимых графах}

Обозначим $M$ множество вершин графа $\Gamma(M)$, в частности, положим $M_{k}=$ $\{1,2, \ldots, k\}$. Через $G(M)$ будем обозначать множество всех неразложимых графов без петель и кратных ребер с помеченными вершинами из множества $M$. Число таких графов обозначим $g(M)=|G(M)|$; в частности, положим $g_{k}=g\left(M_{k}\right)$. Обозначим $G_{0}(M)\left(G_{1}(M)\right)$ множество всех неразложимых графов из $G(M)$ с четным (нечетным) числом ребер; в частности, положим $G_{k i}=G_{i}\left(M_{k}\right)$. Мощности этих множеств обозначим $g_{i}(M)=\left|G_{i}(M)\right|, g_{k i}=\left|G_{k i}\right|$; положим $\Delta g(M)=g_{0}(M)-g_{1}(M), \Delta g_{k}=g_{k 0}-g_{k 1}$.

Лемма 1. Для любого $k \geqslant 2$

$$
\Delta g_{k}=(-1)^{k-1}(k-1) !
$$

Доказательство. Докажем лемму по индукции. При $k=2$ существует только один связный граф, и равенство (2) выполняется. Предположим, что для всех $i \leqslant k$ равенство (2) выполняется, и докажем его справедливость для $k+1$. $\mathrm{Pa-}$ зобьем доказательство на три этапа.

(a) Обозначим $G_{k+1,0}^{\prime}\left(G_{k+1,1}^{\prime}\right)$ множество таких неразложимых графов $\Gamma\left(M_{k+1}\right)$ с четным (нечетным) числом ребер, подграфы $\Gamma\left(M_{k}\right)$ которых также неразложимы, и обозначим $g_{k+1, i}^{\prime}=\left|G_{k+1, i}^{\prime}\right|$. Имеет место равенство

$$
g_{k+1,0}^{\prime}-g_{k+1,1}^{\prime}=-\left(g_{k 0}-g_{k 1}\right)
$$

В самом деле,

$$
\begin{aligned}
g_{k+1,0}^{\prime}-g_{k+1,1}^{\prime}= & \left(\left(\begin{array}{l}
k \\
1
\end{array}\right)+\left(\begin{array}{l}
k \\
3
\end{array}\right)+\ldots\right) g_{k 1}+\left(\left(\begin{array}{l}
k \\
2
\end{array}\right)+\left(\begin{array}{l}
k \\
4
\end{array}\right)+\ldots\right) g_{k 0} \\
& -\left(\left(\begin{array}{l}
k \\
1
\end{array}\right)+\left(\begin{array}{l}
k \\
3
\end{array}\right)+\ldots\right) g_{k 0}-\left(\left(\begin{array}{l}
k \\
2
\end{array}\right)+\left(\begin{array}{l}
k \\
4
\end{array}\right)+\ldots\right) g_{k 1} \\
= & g_{k 1}-g_{k 0} .
\end{aligned}
$$

(b) Пусть $M^{\prime}$ и $M^{\prime \prime}$ - разбиение множества $M_{k}$, т.е.

$$
M_{k}=M^{\prime} \cup M^{\prime \prime}, \quad M^{\prime} \cap M^{\prime \prime}=\varnothing .
$$


Обозначим $G^{\prime \prime}\left(M_{k+1} \mid M^{\prime}, M^{\prime \prime}\right)$ множество всех таких графов $\Gamma\left(M_{k+1}\right)$ с вершинами $M_{k+1}$, что никакая пара вершин $a \in M^{\prime}, b \in M^{\prime \prime}$ не соединена ребром, а подграфы $\Gamma\left(M^{\prime} \cup\{k+1\}\right)$ и $\Gamma\left(M^{\prime \prime} \cup\{k+1\}\right)$ неразложимы. Обозначим $g_{0}^{\prime \prime}\left(M_{k+1} \mid M^{\prime}, M^{\prime \prime}\right) \quad\left(g_{1}^{\prime \prime}\left(M_{k+1} \mid M^{\prime}, M^{\prime \prime}\right)\right)$ число графов из множества $G^{\prime \prime}\left(M_{k+1} \mid M^{\prime}, M^{\prime \prime}\right)$ с четным (нечетным) числом ребер, и

$$
\Delta g^{\prime \prime}\left(M_{k+1} \mid M^{\prime}, M^{\prime \prime}\right)=g_{0}^{\prime \prime}\left(M_{k+1} \mid M^{\prime}, M^{\prime \prime}\right)-g_{1}^{\prime \prime}\left(M_{k+1} \mid M^{\prime}, M^{\prime \prime}\right) .
$$

Тогда

$$
\Delta g^{\prime \prime}\left(M_{k+1} \mid M^{\prime}, M^{\prime \prime}\right)=\Delta g\left(M^{\prime} \cup\{k+1\}\right) \Delta g\left(M^{\prime \prime} \cup\{k+1\}\right) .
$$

Равенство (4) следует из очевидного соотношения

$$
\begin{aligned}
& g_{0}^{\prime \prime}\left(M_{k+1} \mid M^{\prime}, M^{\prime \prime}\right) \\
& \quad=g_{0}\left(M^{\prime} \cup\{k+1\}\right) g_{0}\left(M^{\prime \prime} \cup\{k+1\}\right)+g_{1}\left(M^{\prime} \cup\{k+1\}\right) g_{1}\left(M^{\prime \prime} \cup\{k+1\}\right)
\end{aligned}
$$

и аналогичного соотношения для $g_{1}^{\prime \prime}\left(M_{k+1} \mid M^{\prime}, M^{\prime \prime}\right)$.

(c) В каждом графе $\Gamma\left(M_{k+1}\right)$ из $G\left(M_{k+1}\right)$ выделим вершины 1 и $k+1$. Обозначим $M^{\prime}$ содержащую вершину 1 компоненту подграфа, получающегося из графа $\Gamma\left(M_{k+1}\right)$ при удалении вершины $k+1$ и всех инцидентных ей ребер. Пусть $\left|M^{\prime}\right|=i, M^{\prime \prime}=M \backslash M_{k},\left|M^{\prime \prime}\right|=k-i$. Учитывая пункты (а) и (b) доказательства, в силу индукции при каждом $i$, равном $1,2, \ldots, k$, из равенства (4) получаем, что

$$
\begin{aligned}
\Delta g^{\prime \prime}\left(M_{k+1} \mid M^{\prime}, M^{\prime \prime}\right) & =\Delta g\left(M^{\prime} \cup\{k+1\}\right) \Delta g\left(M^{\prime \prime} \cup\{k+1\}\right) \\
& =-\Delta g\left(M^{\prime}\right) \Delta g\left(M^{\prime \prime} \cup\{k+1\}\right) \\
& =-(-1)^{i-1}(i-1) !(-1)^{k-i}(k-i) !, \quad i=1,2, \ldots, k .
\end{aligned}
$$

Отсюда следует, что

$$
\Delta g_{k+1}=(-1)^{k} \sum_{i=1}^{k}(i-1) !(k-i) !\left(\begin{array}{c}
k-1 \\
i-1
\end{array}\right)=(-1)^{k} k !
$$

Лемма доказана.

\section{3. Основная теорема}

Пусть $J=\left\{j_{1}, \ldots, j_{l}\right\}$ - подмножество множества $M_{m}=\{1,2, \ldots, m\}$. Положим

$$
\xi(J, n)=\sum_{r=1}^{n} \alpha_{r j_{1}} \alpha_{r j_{2}} \ldots \alpha_{r j_{l}}
$$

иногда мы будем использовать сбозначения

$$
\xi\left(\left\{j_{1}, \ldots, j_{l}\right\}, n\right)=\xi_{j_{1}, \ldots, j_{l}}(n) .
$$

Обозначим $K=\left(K_{1}, \ldots, K_{r}\right)$ разбиение множества $M_{m}=\{1,2, \ldots, m\}, k_{i}=\left|K_{i}\right|$; пусть $\mathcal{K}_{p}=\{K\}$ - множество всех разбиений $M_{m}$, у которых $k_{i} \leqslant p, i=1, \ldots, r$. 
Теорема 1. Если әлементы перманента матрицы $A_{n m}=\left\|\alpha_{i j}\right\|$ принадлежсат nолю $\mathrm{GF}(p)$, mo

$$
\operatorname{per}\left(A_{n m}\right)=\sum_{K \in \mathcal{K}_{p}} c(K) \xi\left(K_{1}, n\right) \xi\left(K_{2}, n\right) \ldots \xi\left(K_{r}, n\right),
$$

әде $K=\left(K_{1}, K_{2}, \ldots, K_{r}\right)$ - разбиения мноэсества $M_{m}, k_{i}=\left|K_{i}\right|, i=1, \ldots, r$, $c(K)=(-1)^{m-r}\left(k_{1}-1\right) !\left(k_{2}-1\right) ! \ldots\left(k_{r}-1\right) !$.

Доказательство. Положим

$$
\delta(i, j ; k, l)= \begin{cases}1, & \text { если } i=j, \\ 0, & \text { если } i \neq j .\end{cases}
$$

Равенство (1) можно записать в виде

$$
\operatorname{per}\left(A_{n m}\right)=\sum_{i_{1}=1}^{n} \sum_{i_{2}=1}^{n} \ldots \sum_{i_{m}=1}^{n} \alpha_{i_{1} 1} \alpha_{i_{2} 2} \ldots \alpha_{i_{m} m} \prod_{1 \leqslant k<l \leqslant m}\left(1-\delta\left(i_{k}, i_{l} ; k, l\right)\right) .
$$

Раскрывая скобки в (7), мы получаем $2^{\left(\begin{array}{c}m \\ 2\end{array}\right)}$ сумм, т.е. ровно столько, сколько имеется графов с $m$ помеченными вершинами. Сумму вида

$$
\sum_{i_{1}, \ldots, i_{m}=1}^{n}(-1)^{r} \alpha_{i_{1} 1} \ldots \alpha_{i_{m} m} \delta\left(i_{k_{1}}, i_{l_{1}} ; k_{1}, l_{1}\right) \ldots \delta\left(i_{k_{r}}, i_{l_{r}} ; k_{r}, l_{r}\right)
$$

обозначим $\xi(\Gamma, n)$, где $\Gamma$ - граф с множеством вершин $M_{m}$, в котором вершины $k_{i}$ и $l_{i}, i=1,2, \ldots, r$, соединены ребрами. Если граф $\Gamma$ состоит из связных компонент $\Gamma_{1}, \Gamma_{2}, \ldots, \Gamma_{s}$, то

$$
\xi(\Gamma, n)=\prod_{t=1}^{s}(-1)^{d\left(\Gamma_{t}\right)} \xi\left(v\left(\Gamma_{t}\right), n\right),
$$

где $v\left(\Gamma_{t}\right)$ - множество вершин компоненты $\Gamma_{t}, d\left(\Gamma_{t}\right)$ - количество ребер графа $\Gamma_{t}, \xi\left(v\left(\Gamma_{t}\right), n\right)$ определены формулой (5). В самом деле, если $\left(k_{1}, l_{1}\right), \ldots,\left(k_{q}, l_{q}\right)-$ все ребра компоненты $\Gamma_{t}$, то

$$
\delta\left(i_{k_{1}}, i_{l_{1}} ; k_{1}, l_{1}\right) \ldots \delta\left(i_{k_{q}}, i_{l_{q}} ; k_{q}, l_{q}\right)=1
$$

тогда и только тогда, когда $i_{k_{1}}=i_{l_{1}}, \ldots, i_{k_{q}}=i_{l_{q}}$, а так как граф $\Gamma_{t}$ связен, то отсюда следует, что все индексы $i_{k_{j}}, i_{l_{j}}, j=1,2, \ldots, q$, равны друг другу. Поэтому та часть суммы (8), которая соответствует графу $\Gamma_{t}$ суммируется отдельно и дает

$$
\xi\left(v\left(\Gamma_{t}\right), n\right)=\sum_{i=1}^{n}(-1)^{d\left(\Gamma_{t}\right)} \prod_{k \in v\left(\Gamma_{t}\right)} \alpha_{i k} .
$$

В частности, при $v(\Gamma)=\{i\}$ и $v(\Gamma)=\{i, j\}$ имеем, соответственно, $\xi\left(v\left(\Gamma_{t}\right), n\right)=$ $\xi_{i}(n)$ и $\xi\left(v\left(\Gamma_{t}\right), n\right)=\xi_{i j}(n)$. Отсюда вытекает $(9)$. В равенстве

$$
\operatorname{per}\left(A_{n m}\right)=\sum_{\Gamma} \xi(\Gamma, n),
$$


где суммирование ведется по всем графам на множестве вершин $M_{m}$, выделим часть суммы следующим образюм. Разложим множество $M_{m}$ на $J$ и $M_{m} \backslash J$. Обозначим $H_{m}\left(\Gamma^{\prime}\right)$ множество всех графов $\Gamma$, которые состоят из связной компоненты $\Gamma_{1}$ с вершинами $v\left(\Gamma_{1}\right)=J$ и фиксированного подграфа $\Gamma^{\prime}$ с вершинами $v\left(\Gamma^{\prime}\right)=M_{m} \backslash J$. Для любого $\Gamma \in H_{m}\left(\Gamma^{\prime}\right)$

$$
\xi(\Gamma, n)=\xi(J, n) \xi\left(\Gamma^{\prime}, n\right) .
$$

Рассмотрим часть суммы (11)

$$
\sum_{\Gamma \in H_{m}\left(\Gamma^{\prime}\right)} \xi(\Gamma, n)=\xi\left(\Gamma^{\prime}, n\right) \sum_{\Gamma_{1} \in G(J)} \xi\left(\Gamma_{1}, n\right),
$$

где $G(J)$ - множество связных графов на вершинах $J$. В силу (10)

$$
\xi\left(\Gamma_{1}, n\right)=(-1)^{d\left(\Gamma_{1}\right)} \xi\left(v\left(\Gamma_{1}\right), n\right) .
$$

Отсюда следует, что $\operatorname{per}\left(A_{n m}\right)$ равен сумме типа (6), когда суммирование ведется по всем разбиением $K$ множества $M_{n}$. Но так как $\operatorname{per}\left(A_{n m}\right)$ вычисляется в поле $\mathrm{GF}(p)$, то все разбиения с $k_{i}>p$ дают коэффициенты $(-1)^{k_{i}-1}\left(k_{i}-1\right) !=0$ по модулю $p$, поэтому суммиронание в (6) можно вести только по разбиениям $K \in \mathcal{K}_{p}$. Теорема доказана.

Замечание 1. Полученная формула не связана со случайностью матрицы $A_{n m}$ и позволяет вычислять $\operatorname{per}\left(A_{n m}\right)$ в поле $\mathrm{GF}(p)$. В частности, при $p=3$

$$
\begin{aligned}
& \operatorname{per}\left(A_{n 1}\right)=\xi_{1}(n) \\
& \operatorname{per}\left(A_{n 2}\right)=\xi_{1}(n) \xi_{2}(n)-\xi_{12}(n), \\
& \operatorname{per}\left(A_{n 3}\right)=\xi_{1}(n) \xi_{2}(n) \xi_{3}(n)-\xi_{1}(n) \xi_{23}(n)-\xi_{2}(n) \xi_{13}(n)-\xi_{3}(n) \xi_{12}(n)+2 \xi_{123}(n) .
\end{aligned}
$$

Замечание 2. Теорема 1 справедлива и для случая кольца вычетов по модулю $m$. В этом случае некоторые слагаемые в сумме (6) обратятся в нуль. Например, при $m=6$ в сумму (6) войдут те слагаемые, в которых будут только случайные величины $\xi_{i}(n), \xi_{i j}(n), \xi_{i j k}(n)$, так как в слагаемом с сомножителем $\xi_{i_{1} i_{2} \ldots i_{l}}(n)$ с $l>3$ имеется коэффициент $(l-1) !=0(\bmod 6)$.

\section{4. Лемма об әргоддичности}

Опишем класс случайных матриц $A_{n m}$, для которых будет доказана предельная теорема. Пусть $R^{m}$ - векторное пространство размерности $m$; векторы $\vec{x}=$ $\left(x_{1}, x_{2}, \ldots, x_{m}\right) \in R^{m}$ имеют компоненты $x_{i} \in \mathrm{GF}(p)$. Определим отображение $S$ пространства $R^{m}$ в $R^{s_{m}}$, где $s_{m}==\sum_{k=1}^{p}\left(\begin{array}{c}m \\ k\end{array}\right)$, полагая

$$
\begin{aligned}
S \vec{x}=\left(x_{1}, \ldots, x_{m}, x_{1} x_{2}, x_{1} x_{3}, \ldots, x_{1} x_{m}, x_{2} x_{3}, x_{2} x_{4}, \ldots, x_{m-1} x_{m},\right. \\
\left.x_{1} x_{2} x_{3}, \ldots, x_{m-p+1} x_{x-p+2} \ldots x_{m}\right) .
\end{aligned}
$$

Здесь и ниже предполагается, что величины с неположительными индексами равны нулю. 
Ниже мы будем рассматривать класс случайных матриц $A_{n m}$, у которых строки $\vec{\alpha}_{i}=\left(\alpha_{i 1}, \alpha_{i 2}, \ldots, \alpha_{i m}\right)$ независимы и одинаково распределены. Для любого $\vec{a} \in R^{m}$ обозначим $q(\vec{a})=\mathbf{P}\left\{\vec{\alpha}_{i}=\vec{a}\right\}$. Легко видеть, что распределение $q(\cdot)$ индуцирует распределение $q^{*}(\cdot)$ на $R^{s_{m}}:$ если $\vec{a} \in R^{s_{m}}$ и $\vec{a}=S \vec{a}^{\prime}$, где $\vec{a}^{\prime} \in R^{m}$, то

$$
q^{*}(\vec{a})=\mathbf{P}\left\{S \vec{\alpha}_{i}^{\prime}=\vec{a}\right\}=\mathbf{P}\left\{\vec{\alpha}_{i}=\vec{a}^{\prime}\right\}=q\left(\vec{a}^{\prime}\right)
$$

В остальных случаях $q^{*}(\vec{a})=0$. Обозначим $\mathcal{A}=\left\{\vec{a} \in R^{s_{m}}: q^{*}(\vec{a})>0\right\}$ и $L(\mathcal{A})$ линейную оболочку $\mathcal{A}$ в $R^{s_{m}}$.

Лемма 2. Пусть в случайной матриче $A_{n m}$ строки $\vec{\alpha}_{i}=\left(\alpha_{i 1}, \ldots, \alpha_{i m}\right)$ независимы и одинаково распределены. Для того, чтобы вектор $\vec{\xi}(n) \in R^{s_{m}}$, составленный из компонент $\xi_{i}(n), \xi_{i j}(n), \xi_{i j k}(n), \ldots, \xi_{m-p+1, m-p+2, \ldots, m}(n)$, определенмых (5), сходился по распределению $к$ вектору

$$
\vec{\xi}=\left(\xi_{1}, \xi_{2}, \ldots, \xi_{m}, \xi_{12}, \xi_{13}, \ldots, \xi_{1 m}, \xi_{23}, \ldots, \xi_{2 m}, \ldots, \xi_{m-p+1, m-p+2, \ldots, m}\right) \in R^{s_{m}}
$$

компоненты которого независимы и равномерно распределены на $\mathrm{GF}(p)$, необходимо и достаточно, чтобы выполнялись следующие два условия:

(1) $L(\mathcal{A})=R^{s_{m}}$

(2) $\exists k \neq 0(\bmod p)$ maxoe, чmo

$$
\sum_{i=1}^{k} \vec{a}(i)=\overrightarrow{0} \in R^{s_{m}}, \quad \vec{a}(i) \in \mathcal{A}, \quad i=1,2, \ldots, k
$$

Доказательство. Из (5) следует, что

$$
\vec{\xi}(n)=\sum_{r=1}^{n} S \vec{\alpha}_{r}
$$

где $S \vec{\alpha}_{r}$ независимы и одинаково распределены с распределением

$$
q^{*}(\vec{a})=\mathbf{P}\left\{S \vec{\alpha}_{r}=\vec{a}\right\}
$$

которое задано формулой (12) с помощью распределения $q(\cdot)$. Последовательность $\vec{\xi}(n)$ образует цепь Маркова с $p^{s_{m}}$ состояниями, представляющими собой все элементы из $R^{s_{m}}$. Переходная вероятность $p(\vec{a}, \vec{b})$ цепи Маркова $\vec{\xi}(n)$ определяется равенством

$$
p(\vec{a}, \vec{b})=\mathbf{P}\{\vec{\xi}(n+1)=\vec{b} \mid \vec{\xi}(n)=\vec{a}\}=q^{*}(\vec{b}-\vec{a}), \quad \vec{a}, \vec{b} \in R^{s_{m}}
$$


Так как

$$
\sum_{\vec{a} \in R^{s_{m}}} p(\vec{a}, \vec{b})=\sum_{\vec{a} \in R^{s_{m}}} q(\vec{b}-\vec{a})=1
$$

переходная матрица $\|p(\vec{a}, \vec{b})\|$ дважды стохастическая. Если мы докажем, что цепь Маркова $\vec{\xi}(n)$ эргодическая, то ее предельное распределение будет равномерным, т.е. для всех $\vec{a} \in R^{s_{m}}$

$$
\lim _{n \rightarrow \infty} P\{\vec{\xi}(n)=\vec{a}\}=\frac{1}{p^{s_{m}}}
$$

Отсода вытекает утверждение леммы. Докажем әргодичность цепи. Из (13) следует, что переходная вероятность за $t$ пагов $p^{(t)}(\vec{a}, \vec{b})$ будет положительной тогда и только тогда, когда найдутся векторы $\vec{a}(k) \in R^{s_{m}}, k=1, \ldots, t$, такие, что $p^{*}(\vec{a}(k))>0$, т.е. $\vec{a}(k) \in \mathcal{A}$, и

$$
\vec{b}=\vec{a}+\sum_{k=1}^{t} \vec{a}(k) .
$$

Поэтому для любых $\vec{a}, \vec{b} \in R^{s_{m}}$ найдется такое $t$, что $p^{(t)}(\vec{a}, \vec{b})>0$ тогда и только тогда, когда выполнено условие 1 леммы. Условие 2 леммы выполняется тогда и только тогда, когда цепь $\vec{\xi}(n)$ апериодична. Обозначим $N_{0}$ множество тех $t \geqslant 1$, для которых $p^{(t)}(\overrightarrow{0}, \overrightarrow{0})>0$. В силу условия 2 леммы и равенства (14) $k \in N_{0}$. Число $p$ также принадлежит $N_{0}$, так как $p \vec{a}=\overrightarrow{0}$ для любого $\vec{a} \in \mathcal{A}$. Поэтому общий наибольший делитель чисел из $N_{0}$ равен 1 , т.е. цепь апериодична. Наоборот, если цепь апериодична, эо условие 2 должно быть выполнено, иначе у цепи будет период $p$. Лемма доказана.

Следствие 1. Если распределекие $q(\vec{a})=\mathbf{P}\{\vec{\alpha}=\vec{a}\}$ таково, что өсе $q(\vec{a})>0$, то условия леммы 2 өыполияотся, $u$, следовательно, $\vec{\xi}(n)$ сходится по распределенио $к$ предельному вехтору $\vec{\xi} \in R^{s_{m}}$ с кезависимыми и равномерно распределенжыми компонентами. В час:тности, это утверхсдение справедливо, если өсе әлементы $\alpha_{i j}$ матричы $A_{n m}$ мезаөисимы $u \mathbf{P}\left\{\alpha_{i j}=a\right\}=p_{j}>0$ для лиобого $a=0,1,2, \ldots, p-1$.

Замечание 3. Если нарушено условие 1, а условие 2 выполнено, то предельное распределение $\vec{\xi}$ существует и равномерно на некотором подпространстве $L(\mathcal{A}) \subset R^{s_{m}}$ меньшей размерности. В этом случае некоторые линейные комбинации компонент вектора $\vec{\xi}$ равны нулю. Например, если $m=2$ и $q((1,2))=q((0,1))=q((0,0))=1 / 3$, то при $p>2 L(\mathcal{A})$ состоит из векторов $\vec{x}=\left(x_{1}, x_{2}, x_{12}\right) \in R^{3}$, у которых $x_{12}=2 x_{1}$. Поэтому предельный вектор $\vec{\xi}=\left(\xi_{1}, \xi_{2}, \xi_{12}\right)$ имеет независимые компоненты $\xi_{1}, \xi_{2}$ с $\mathbf{P}\left\{\xi_{1}=a\right\}=\mathbf{P}\left\{\xi_{2}=a\right\}=1 / p$ для $a=0, \ldots, p-1$ и $\mathbf{P}\left\{\xi_{12}=2 \xi_{1}\right\}=1$. 


\section{5. Предельная теорема}

С помощью леммы 2 можно доказать предельную теорему для распределения $\operatorname{per}\left(A_{n m}\right)$ при $n \rightarrow \infty$. Воспользуемся обозначениями $\S 3$. Пусть $\mathcal{K}_{p}-$ множество всех разбиений $K=\left(K_{1}, K_{2}, \ldots, K_{r}\right), r \geqslant 1$, множества $M_{m}=\{1,2, \ldots, m\}$, для которых $k_{i}=\left|K_{i}\right| \leqslant p, i=1, \ldots, r$. Для любого подмножества $A \subseteq M_{m}$ обозначим $\xi(A)$ равномерно распределенную на $\mathrm{GF}(p)$ случайную величину. Если $A=$ $\left\{i_{1}, i_{2}, \ldots, i_{r}\right\}, i_{1}<i_{2}<\ldots<i_{r}$, то будем обозначать также

$$
\xi(A)=\xi_{i_{1} i_{2} \ldots i_{r}} .
$$

Будем полагать, что все случайные величины $\xi(A), A \subseteq M_{m}$, независимы. Обозначим

$$
\xi(K)=\xi\left(K_{1}\right) \xi\left(K_{2}\right) \ldots \xi\left(K_{r}\right),
$$

где разбиение $K=\left(K_{1}, K_{2}, \ldots, K_{r}\right), r \geqslant 1$.

Теорема 2. В условиях леммы 2 существуют предельные вероятности вида

$$
\lim _{n \rightarrow \infty} \mathbf{P}\left\{\operatorname{per}\left(A_{n m}\right)=k\right\}=p_{k}=\rho_{m} \delta_{k 0}+\left(1-\rho_{m}\right) / p, \quad k=0,1, \ldots, p-1,
$$

әде $\delta_{k 0}$ - символ Кронекера. Это распределение совпадает с распределением случайной величины

$$
\eta_{m}=\sum_{K \in \mathfrak{K}_{p}} \xi(K)
$$

Дохазательство. Из формулы (6), теоремы 1 и леммы 2 следует, что предельное распределение $\operatorname{per}\left(A_{n m}\right)$ при $n \rightarrow \infty$ совпадает с распределением случайной величины

$$
\sum_{K \in \mathcal{K}_{p}} c(K) \xi\left(K_{1}\right) \xi\left(K_{2}\right) \ldots \xi\left(K_{r}\right) .
$$

Поскольку $\xi\left(K_{i}\right)$ независимы и равномерно распределены, то их распределение совпадает с распределением $(-1)^{k_{i}-1}\left(k_{i}-1\right) ! \xi\left(K_{i}\right)$. Так как распределение случайной величины (16) инвариантно относительно ее умножения на любое $a \in \mathrm{GF}(p), a \neq 0$, отсюда следует утверждение теоремы.

В работе [1] теорема 2 доказана при $p=2$. Там же на основе этой теоремы вычислено предельное распределение $\operatorname{per}\left(A_{n m}\right)$ при $n \rightarrow \infty$ и любом $m$. При $p \geqslant 3$ эти предельные вероятности удается вычислить только для $1 \leqslant m \leqslant p+2$.

Теорема 3. Параметр $\rho_{m}$ в вероятностях (15) имеет следующий вид:

$$
\begin{array}{lll}
\rho_{m}=0 & \text { npu } 1 \leqslant m \leqslant p \\
\rho_{m}=1 / p^{\beta}, & \beta=2^{p}-1, & \text { npu } m=p+1 \\
\rho_{m}=1 / p^{\gamma}, & \gamma=2^{p+1}-p-3, & \text { npu } m=p+2 .
\end{array}
$$


Доказательство. Пусть множество $M \subseteq M_{m}=\{1,2, \ldots, m\}$. Если $M=$ $\left\{i_{1}, i_{2}, \ldots, i_{r}\right\}$, то, согласно введенному ранее обозначению, $\xi(M)=\xi_{i_{1} i_{2} \ldots i_{r}}$. Введем случайные величины $\varphi_{r}(M)$, где $r=|M|$, следующим рекуррентным соотношением:

$$
\varphi_{r}(M)=\sum_{J \in D_{l}} \xi(J) \varphi_{r-|J|}(M \backslash J)
$$

где $D_{l}$ - множество таких подмножеств $J \subseteq M$, которые удовлетворяют следующим условиям:

$$
l \in J \subseteq M, \quad|J| \leqslant p .
$$

Полагая $\varphi_{0}(\varnothing)=1$, последовательно получаем из (17)

$$
\begin{aligned}
\varphi_{1}(\{i\}) & =\xi_{i}, \\
\varphi_{2}(\{i, j\}) & =\xi_{i j}+\xi_{i} \xi_{j} \\
\varphi_{3}(\{i, j, k\}) & =\xi_{i j k}+\xi_{i j} \xi_{k}+\xi_{i k} \xi_{j}+\xi_{j k} \xi_{i}+\xi_{i} \xi_{j} \xi_{k}
\end{aligned}
$$

и т. д. Случайную величину $\eta_{m}$ в $(16)$ можно записать с помощью разложения (17) следующим образом:

$$
\eta_{m}=\sum_{J \in D_{1}} \xi(J) \varphi_{m-|J|}\left(M_{m} \backslash J\right)
$$

При $m \leqslant p$ в разложении (18) имеется слагаемое $\xi\left(M_{m}\right)=\xi_{12 \ldots m}$, поэтому случайная величина $\eta_{m}$ равномерно распределена, т.е. $\rho_{m}=0$. Если $m=p+1$, то нетрудно показать, что в сумме (18) все случайные величины $\xi(J)$ и $\varphi_{m-|J|}\left(M_{m} \backslash J\right)$ независимы и равномерно распределены, а число слагаемых равно $\beta=2^{p}-1$. Событие

$$
B=\bigcap_{J \in D_{1}}\left\{\varphi_{m-|J|}\left(M_{m} \backslash J\right)=0\right\}
$$

имеет вероятность $\mathrm{P}(B)=1 / p^{\beta}$. Для любого $a \neq 0, a \in \mathrm{GF}(p)$, получаем, что

$$
\begin{aligned}
\mathbf{P}\left\{\eta_{m}=a\right\} & =\mathbf{P}\{B\} \mathbf{P}\{\eta=a \mid B\}+\mathbf{P}\{\bar{B}\} \mathbf{P}\{\eta=a \mid \bar{B}\} \\
& =\left(1-1 / p^{\beta}\right) / p .
\end{aligned}
$$

Если $m=p+2$, то в (18) все случайные величины $\xi(J)$ и $\varphi_{m-|J|}\left(M_{m} \backslash J\right)$, кроме $\varphi_{m-1}\left(M_{m} \backslash\{1\}\right)$, независимы и равномерно распределены, а число слагаемых в (18) с $|J|>1$ равно $\gamma=2^{p+1}-p-3$. Обозначим событие

$$
B_{r}=\bigcap_{\substack{J \in D_{1} \\|J| \geqslant m-r}}\left\{\varphi_{m-|J|}\left(M_{m} \backslash J\right)=0\right\}
$$

Докажем по индукции, что из события $B_{r}, 1 \leqslant r \leqslant p$, следует, что для любых $2 \leqslant j_{1}<j_{2}<\ldots<j_{r} \leqslant m$

$$
\xi_{j_{1} j_{2} \ldots j_{r}}=(-1)^{r-1}(r-1) ! \xi_{j_{1}} \xi_{j_{2}} \ldots \xi_{j_{r}} .
$$

При $r=2$ из $\varphi_{2}(\{i, j\})=\xi_{i j}+\xi_{i} \xi_{j}=0$ следует, что $\xi_{i j}=-\xi_{i} \xi_{j}$, и (19) выполняется. Предположим, что при выполнении события $B_{r_{0}}, r_{0}<p$, выполняются все 
равенства (19) для $r \leqslant r_{0}$. Докажем, что тогда (19) справедливо и при $r=r_{0}+1$. Пусть $M=\left\{j_{1}, j_{2}, \ldots, j_{r_{0}+1}\right\}$; из формулы $(17)$ находим, что

$$
\varphi_{r_{0}+1}(M)=\sum_{J \in D_{j_{r_{0}+1}}} \xi(J) \varphi_{r_{0}+1-|J|}(M \backslash J)
$$

Из события $B_{r_{0}+1}$ следует событие $B_{r_{0}}$. Поэтому при $1 \leqslant|J| \leqslant r_{0}-1$ все $\varphi_{r_{0}+1-|J|}(M \backslash J)=0$, и (20) превратится в равенство

$$
\varphi_{r_{0}+1}(M)=\xi_{j_{1} j_{2} \ldots j_{r_{0}+1}}+\sum_{k=1}^{r_{0}} \xi_{j_{1} \ldots j_{k-1} j_{k+1} \ldots j_{r_{0}+1}} \xi_{j_{k}} .
$$

Из $B_{r_{0}+1}$ следует, что $\varphi_{r_{0}+1}(M)=0$; поэтому. равенство (19) выполняется и при $r=r_{0}+1 \leqslant p$. Таким образом, если имеет место событие $B_{m-2}$, то равенства (19) выполняются для всех $2 \leqslant r \leqslant p$. Покажем теперь, что события $B_{m-1}$ и $B_{m-2}$ равносильғы, т.е. из равенства $\varphi_{m-|J|}(M \backslash J)=0$ при всех $J \in D_{1},|J| \geqslant$ 2 , следует, что $\varphi_{m-1}\left(M_{m} \backslash\{1\}\right)=0$. Рассуждения, аналогичные тем, которые привели к доказательству (19), имеют следствием следующее равенство в GF (p):

$$
\varphi_{m-1}\left(M_{m} \backslash\{1\}\right)=\sum_{k=1}^{p}(-1)^{p-1}(p-1) ! \xi_{2} \xi_{3} \ldots \xi_{m}=0 .
$$

Таким образом, при всех $a \in \mathrm{GF}(p), a \neq 0$,

$$
\begin{aligned}
\mathbf{P}\left\{\eta_{m}=a\right\} & =\mathbf{P}\left\{\bar{B}_{m-1}\right\} \mathbf{P}\left\{\eta_{m}=a \mid \bar{B}_{m-1}\right\}+\mathbf{P}\left\{B_{m-1}\right\} \mathbf{P}\left\{\eta_{m}=a \mid B_{m-1}\right\} \\
& =\mathbf{P}\left\{\bar{B}_{m-2}\right\} \mathbf{P}\left\{\eta_{m}=a \mid \bar{B}_{m-2}\right\}=\left(1-1 / p^{\gamma}\right) / p
\end{aligned}
$$

откуда следует, что $\rho_{p+2}=1 / p^{\gamma}$. Теорема доказана.

При $p=3$ с помощью преобразований

$$
\begin{aligned}
\xi_{i j}^{\prime} & =\xi_{i j}+\xi_{i} \xi_{j}, \\
\xi_{i j k}^{\prime} & =\xi_{i j k}+\xi_{i} \xi_{j k}+\xi_{j} \xi_{i k}+\xi_{k} \xi_{i j}+\xi_{i} \xi_{j} \xi_{k}
\end{aligned}
$$

случайную величину $\eta_{6}$ в (18) можно представить в виде суммы двух независимых случайных величин

$$
\eta_{6}=\eta^{\prime}+\eta^{\prime \prime}
$$

где

$$
\begin{aligned}
& \eta^{\prime}=\xi_{123}^{\prime} \xi_{456}^{\prime}+\xi_{124}^{\prime} \xi_{356}^{\prime}+\xi_{125}^{\prime} \xi_{346}^{\prime}+\xi_{126}^{\prime} \xi_{345}^{\prime}+\xi_{134}^{\prime} \xi_{256}^{\prime}+\xi_{135}^{\prime} \xi_{246}^{\prime}+\xi_{136}^{\prime} \xi_{245}^{\prime} \\
& \quad+\xi_{145}^{\prime} \xi_{236}^{\prime}+\xi_{146}^{\prime} \xi_{235}^{\prime}+\xi_{156}^{\prime} \xi_{234}^{\prime} \\
& \begin{aligned}
\eta^{\prime \prime}= & \xi_{12}^{\prime}\left(\xi_{34}^{\prime} \xi_{56}^{\prime}+\xi_{35}^{\prime} \xi_{46}^{\prime}+\xi_{36}^{\prime} \xi_{45}^{\prime}\right)+\xi_{13}^{\prime}\left(\xi_{24}^{\prime} \xi_{56}^{\prime}+\xi_{25}^{\prime} \xi_{46}^{\prime}+\xi_{26}^{\prime} \xi_{45}^{\prime}\right) \\
& +\xi_{14}^{\prime}\left(\xi_{23}^{\prime} \xi_{56}^{\prime}+\xi_{25}^{\prime} \xi_{36}^{\prime}+\xi_{26}^{\prime} \xi_{35}^{\prime}\right)+\xi_{15}^{\prime}\left(\xi_{23}^{\prime} \xi_{46}^{\prime}+\xi_{24}^{\prime} \xi_{36}^{\prime}+\xi_{26}^{\prime} \xi_{34}^{\prime}\right) \\
& +\xi_{16}^{\prime}\left(\xi_{23}^{\prime} \xi_{45}^{\prime}+\xi_{24}^{\prime} \xi_{35}^{\prime}+\xi_{25}^{\prime} \xi_{34}^{\prime}\right) .
\end{aligned}
\end{aligned}
$$

Случайная величина $\eta^{\prime}$ имеет распределение вида (15) с $\rho^{\prime}=1 / 3^{10}$. На ПК вычислено значение $\rho^{\prime \prime}=1173 / 3^{10}$ распределения $\eta^{\prime \prime}$. Таким образом, распределение $\eta_{6}$ определяется параметром $\rho_{6}=\rho^{\prime} \rho^{\prime \prime}=1173 / 3^{20}$. 


\section{Список литературы}

1. Ляпков Л. А., Севастьянов Б. А. Распределение вероятностей перманента случайной булевой матрицы. Дискретная математика (1990) 2, №2, 138-144.

Статья поступила 17.10.95. 